Article

\title{
On Thermodynamics Problems in the Single-Phase-Lagging Heat Conduction Model
}

\author{
Shu-Nan Li and Bing-Yang Cao * \\ Key Laboratory for Thermal Science and Power Engineering of Ministry of Education, \\ Department of Engineering Mechanics, Tsinghua University, Beijing 100084, China; \\ lishunan16@mails.tsinghua.edu.cn \\ * Correspondence: caoby@tsinghua.edu.cn; Tel.: +86-10-6279-4531
}

Academic Editor: Brian Agnew

Received: 10 October 2016; Accepted: 2 November 2016; Published: 9 November 2016

\begin{abstract}
Thermodynamics problems for the single-phase-lagging (SPL) model have not been much studied. In this paper, the violation of the second law of thermodynamics by the SPL model is studied from two perspectives, which are the negative entropy production rate and breaking equilibrium spontaneously. The methods for the SPL model to avoid the negative entropy production rate are proposed, which are extended irreversible thermodynamics and the thermal relaxation time. Modifying the entropy production rate positive or zero is not enough to avoid the violation of the second law of thermodynamics for the SPL model, because the SPL model could cause breaking equilibrium spontaneously in some special circumstances. As comparison, it is shown that Fourier's law and the CV model cannot break equilibrium spontaneously by analyzing mathematical energy integral.
\end{abstract}

Keywords: Fourier's law; Cattaneo-Vernotte model; single-phase-lagging model; laws of thermodynamics

\section{Introduction}

Fourier's law of heat conduction, which shows the connection between temperature gradient and heat flux, is often used to describe normal heat conduction problems:

$$
q+\lambda \nabla T=0,
$$

where $q$ is the heat flux, $\lambda$ is the thermal conductivity and $T$ is the temperature. In recent years, limitations of Fourier's law have been revealed [1-6] such as the fact that Fourier's law predicts an unphysically infinite speed of heat perturbation propagation, and it fails to characterize supertransient and high heat flux processes well. Several modified heat conduction models have been proposed to overcome these limitations. The Cattaneo-Vernotte (CV) model $[7,8]$ is the most typical one:

$$
q+\tau \frac{\partial q}{\partial t}+\lambda \nabla T=0
$$

where $\tau$ is the thermal relaxation time. The heat conduction equations of the CV model is:

$$
\frac{\partial T}{\partial t}+\tau \frac{\partial^{2} T}{\partial t^{2}}=\frac{\lambda}{\rho c_{V}} \nabla^{2} T
$$

where $\rho$ is the mass density and $c_{V}$ is the specific heat. Equation (3) is a hyperbolic heat conduction equation, which predicts wave-like transport in heat conduction processes, called thermal wave. In thermodynamics, researchers have discussed the violation of the second law of thermodynamics by 
the CV model [9-14]. It is found that the CV model could lead to a negative entropy production rate in the framework of classical irreversible thermodynamics. This problem can be overcome by extended irreversible thermodynamics [15], where the definitions of thermodynamics could be extended and appropriate constitutive assumptions are proposed. There are many non-Fourier heat conduction models which have close connection with the CV one, and the single-phase-lagging (SPL) model [16] is a very classical example of them:

$$
q(x, y, z, t+\tau)+\lambda \nabla T(x, y, z, t)=0
$$

The heat conduction equation of the SPL model is

$$
\frac{\partial T(x, y, z, t+\tau)}{\partial t}=\frac{\lambda}{\rho c_{V}} \nabla^{2} T(x, y, z, t-\tau)
$$

The SPL model can reduce to the CV model if a first-order Taylor series approximation is taken for $q(x, y, z, t+\tau)$, which is expressed as:

$$
q(x, y, z, t+\tau) \approx q(x, y, z, t)+\tau \frac{\partial q(x, y, z, t)}{\partial t}
$$

When the approximation in Equation (6) is applied to Equation (4), the SPL model will reduce to the CV model. Because of this approximation relationship between them, the SPL model is sometimes considered as an extension or explanation of the CV one [17-21]. In addition, this approximation also has some defects [22]. However, although there are many thermodynamics discussions about the CV model, thermodynamics problems for the SPL model have not been studied much. There are also other non-Fourier heat conduction models. The Jeffrey model [2] is an extension of the CV model, which can be considered as a linear superposition of Fourier's law and the CV model. The two-temperature model [23] is used for metals by regarding the interactions of electrons and phonons. The Guyer-Krumhansl (GK) model [24] is a very classical model for pure phonon heat conduction. The dual-phase-lagging model [25] adds the influence of temperature lagging on the basis of the single-phase-lagging model. Thermomass theory [26-29] under extreme conditions applies relativity and mass-energy equations to non-Fourier heat conduction.

In this paper, the violation of the second law of thermodynamics by the SPL model is studied from two perspectives, which are the negative entropy production rate and the spontaneous breaking of equilibrium. Compared with the CV model, the methods for the SPL model to avoid the negative entropy production rate are proposed from two perspectives, which are extended irreversible thermodynamics and the thermal relaxation time. Modifying the entropy production rate to a positive or zero value is not enough to avoid the violation of the second law of thermodynamics for the SPL model, because the SPL model could cause spontaneous equilibrium breaking in some special circumstances. As comparison, it is shown why Fourier's law and the CV model cannot break equilibrium spontaneously by analyzing the mathematical energy integral.

\section{Entropy Production Rate}

In classical irreversible thermodynamics (CIT) [15], the entropy production rate $\dot{S}$ for heat conduction is calculated as:

$$
\dot{S}=-\frac{q \cdot \nabla T}{T^{2}}
$$

For Fourier's law, we have:

$$
\dot{S}_{F}=-\frac{q \cdot \nabla T}{T^{2}}=\frac{q^{2}}{\lambda T^{2}} \geq 0
$$


which satisfies the second law of thermodynamics. The negative entropy production rate problem caused by the CV model has been discussed [9-12], and we will discuss this problem for the SPL model. From Equation (4), we can obtain:

$$
\dot{S}_{S P L}=\frac{q(x, y, z, t) \cdot q(x, y, z, t+\tau)}{\lambda T^{2}}
$$

which is not necessarily positive or zero. Next, we will provide a simple example for the negative entropy production rate problem caused by the SPL model. Consider a one-dimensional problem in $0 \leq x \leq l$, where the initial condition is taken as $\left.T\right|_{t=0}=T_{0}\left(2+\sin \frac{\pi x}{l}\right)$, the boundary conditions are taken as $\left.T\right|_{x=0, l}=T_{0}$ and the physical properties satisfy $\frac{\lambda \tau \pi}{c_{V} l^{2}}=\frac{1}{2}$. For this problem, if we use the single-phase-lagging model, we can get the classical solution:

$$
T_{1}(x, t)=T_{0}\left(2+\cos \frac{\pi t}{2 \tau} \sin \frac{\pi x}{l}\right)
$$

From the temperature field, we can get the heat flux field:

$$
q_{1}(x, t)=-\lambda T_{0} \frac{\pi}{l} \sin \frac{\pi t}{2 \tau} \cos \frac{\pi x}{l}
$$

Then we can obtain the entropy production rate $\dot{S}_{1}$ :

$$
\dot{S}_{1}=-\frac{q_{1} \nabla T_{1}}{T_{1}^{2}}=\frac{\lambda \pi^{2} T_{0}^{2}}{l^{2} T_{1}^{2}} \cos ^{2} \frac{\pi x}{l} \sin \frac{\pi t}{2 \tau} \cos \frac{\pi t}{2 \tau}=\frac{\lambda \pi^{2} T_{0}^{2}}{2 l^{2} T_{1}^{2}} \cos ^{2} \frac{\pi x}{l} \sin \frac{\pi t}{\tau}
$$

It is not difficult to find that the above entropy production rate could be negative. For example, when $t=\tau$ and $x=\frac{l}{4}, \dot{S}_{1}=\frac{\lambda \pi^{2} T_{0}^{2}}{4 l^{2} T_{1}^{2}}<0$. Therefore, in the framework of classical irreversible thermodynamics, the SPL model also causes a negative entropy production rate. In the framework of extended irreversible thermodynamics [15], where the definitions of thermodynamics could be extended, the negative entropy production rate problem caused by the CV model can be avoided. That is because appropriate constitutive assumption for entropy could influence the evaluation of the entropy production rate and make it always positive or zero. For example, for the CV model, the constitutive assumption for entropy could be taken as [15]:

$$
S=S(u, q)=S_{e q}(u)-\frac{\tau}{2 \rho \lambda T^{2}} q \cdot q
$$

where $u$ is the internal energy and $S_{e q}(u)$ is the entropy in equilibrium. Then the entropy production rate $\dot{S}$ is calculated as:

$$
\dot{S}=q \cdot\left[-\frac{\nabla T}{T^{2}}-\frac{\tau}{\lambda T^{2}} \frac{\partial q}{\partial t}\right]
$$

Substituting the CV model into Equation (14) leads to:

$$
\dot{S}=\frac{q \cdot q}{\lambda T^{2}}
$$

which is positive or zero. Similarly, for the SPL model, we can also make the entropy production rate always positive or zero by extending the definitions. For example, we can extend entropy flux as $\frac{q(t+\tau)}{T}$ and from this extension, we have an extended calculation of entropy $S=c_{V} \int \frac{1}{T} \frac{\partial T(t+\tau)}{\partial t} d t$. This extension of entropy flux means that for relaxation heat conduction, entropy flux at $t$ is not determined by heat flux at $t$ but determined by heat flux at $(t+\tau)$. Then the calculation of the entropy production rate $\dot{S}$ turns to: 


$$
\dot{S}=-\frac{q(t+\tau) \cdot \nabla T}{T^{2}}
$$

Substituting the SPL model into Equation (16), we can obtain:

$$
\dot{S}=\frac{q(t+\tau) \cdot q(t+\tau)}{\lambda T^{2}}
$$

which is positive or zero.

\section{Thermal Relaxation Time}

There is another perspective about avoiding the negative entropy production rate for the SPL model. The term $q$ must be continuous in time because there is a time differential $\frac{\partial}{\partial t}$ in the heat conduction equation. Based on the continuity of $q$, we can obtain that there is a $\tau_{S}>0$, and when $\tau<\tau_{S}, q(x, y, z, t) q(x, y, z, t+\tau) \geq 0$. Therefore, the entropy production rate will be positive or zero as long as the relaxation time is small enough, which means that reducing the thermal relaxation time (larger than zero) could be another way for avoiding the negative entropy production rate. As an example, we consider again the heat conduction problem in Section 2, where the initial condition is taken as $\left.T\right|_{t=0}=T_{0}\left(2+\sin \frac{\pi x}{l}\right)$, the boundary conditions are taken $\left.T\right|_{x=0, l}=T_{0}$ and the physical properties satisfy $\frac{\lambda \tau \pi}{c_{V} l^{2}}=\frac{1}{2}$. If we reduce the thermal relaxation time $\tau$ to $\tau^{\prime}$, where $\tau^{\prime}=\frac{\tau}{\pi \sqrt{e}}$, and keep the other physical properties, the initial and boundary conditions unchanged, the classical solution for this new heat conduction problem is:

$$
T_{2}(x, t)=T_{0}\left(2+e^{-\frac{t}{2 \tau^{\prime}}} \sin \frac{\pi x}{l}\right)
$$

Then we can obtain the entropy production rate $\dot{S}_{2}$ :

$$
\dot{S}_{2}=-\frac{q_{2} \nabla T_{2}}{T_{2}^{2}}=\frac{\lambda \sqrt{e} e^{-\frac{t}{\tau^{\prime}}} \pi^{2} T_{0}^{2}}{l^{2} T_{2}^{2}} \cos ^{2} \frac{\pi x}{l} \geq 0
$$

From Equation (19) we can find that when $\tau$ is reduced to $\tau^{\prime}$, the entropy production rate changes from $\dot{S}_{1}$, which could be negative, to $\dot{S}_{2}$, which is always positive or zero. In this problem, reducing relaxation time changes the form of the solution and then, the form of the entropy production rate is changed too, which avoids the negative entropy production rate. Therefore, for this problem, a small enough relaxation time (larger than zero) can be another way to avoid the negative entropy production rate. However, reducing the relaxation time may not be effective in some cases, because $\tau<\tau^{\prime}$ could be impossible no matter how small the relaxation time is. In fact, $\tau_{S}$ is determined by the initial and boundary conditions, which can be expressed as $\tau_{S}=\tau_{S}\left(\left.T\right|_{\Gamma},\left.T\right|_{t=0}\right)$. Then if the initial or boundary conditions are influenced by the relaxation time (such as $\left.T\right|_{\Gamma}=\left.T\right|_{\Gamma}(t, \tau),\left.T\right|_{t=0}=$ $\left.\left.T\right|_{t=0}(x, y, z, \tau)\right), \tau_{S}$ will also be influenced by the relaxation time, which means $\tau_{S}=\tau_{S}(\tau)$. Therefore, as a function of $\tau$, it is not necessary that $\tau_{S}(\tau)$ satisfies $\tau<\tau_{S}(\tau)$. Next, we will provide a simple example for this problem, where $\left.T\right|_{\Gamma}=\left.T\right|_{\Gamma}(t, \tau)$. Consider a one-dimensional problem in $0 \leq x \leq l$, where the initial condition is taken as $\left.T\right|_{t=0}=2 T_{0}$, and the boundary conditions are taken as $\left.T\right|_{x=0}=T_{0}\left(2+\sin \frac{3 \pi t}{2 \tau}\right)$ and $\left.T\right|_{t=l}=T_{0}\left(2+\sin \frac{3 \pi t}{2 \tau} e^{-l \sqrt{\frac{3 \pi \rho c_{V}}{2 \tau \lambda}}}\right)$. The classical solution for this problem is:

$$
T_{3}(x, t)=T_{0}\left(2+\sin \frac{3 \pi t}{2 \tau} e^{-x \sqrt{\frac{3 \pi \rho c_{V}}{2 \tau \lambda}}}\right)
$$


Different from the problem in Section 2, there are no conditions for the physical properties. Therefore, the form of the solution will not be changed by reducing the relaxation time and so will the entropy production rate. From Equation (20), we can obtain the entropy production rate $\dot{S}_{3}$ :

$$
\dot{S}_{3}=-\frac{q_{3} \nabla T_{3}}{T_{3}^{2}}=\frac{3 \pi \rho c_{V} e^{-2 x \sqrt{\frac{3 \pi \rho c_{V}}{2 \tau \lambda}}} T_{0}^{2}}{4 \tau T_{3}^{2}} \sin \frac{3 \pi t}{\tau}
$$

It is not difficult to find that Equation (14) could be negative. For example, when $t=\frac{\tau}{2}$ and $x=0, \dot{S}_{3}=-\frac{3 \pi \rho c_{V} T_{0}^{2}}{4 \tau T_{3}^{2}}<0$. Because the form of the entropy production rate is always expressed as Equation (21) no matter how small the relaxation time is, reducing the relaxation time cannot avoid the negative entropy production rate. Compared with the problem in Section 2, where the initial and boundary conditions are not influenced by $\tau$, in this problem, reducing the relaxation time cannot change the form of the solution, because the change of the relaxation time is offset by the boundary conditions $\left.T\right|_{\Gamma}=\left.T\right|_{\Gamma}(t, \tau)$, which will also change as the relaxation time changing. The thermal relaxation time is usually discussed in the CV model, and the Taylor series approximation expressed by Equation (6) seems that the SPL model has the same relaxation time as the CV model. However, the physical meanings of the thermal relaxation time in the two models are quite different. For the SPL model, the heat flux only depends on the previous instantaneous temperature gradient $\tau$, but for the CV model, from Equation (2), it is not difficult to find that the temperature gradient at a certain moment must influence the heat flux at the same moment. Therefore, although the Taylor series approximation expressed by Equation (6) is often established between the two models, their thermal relaxation times have different physical meanings. For the SPL model, the thermal relaxation means that the speed of the heat flux response to temperature gradient is not infinite. For the CV model, whose heat conduction equation is a hyperbolic equation and predicts wave-like transport, the thermal relaxation means that the speed of temperature perturbation itself is not infinite.

\section{Spontaneous Equilibrium}

Besides the positive or zero entropy production rate, the second law of thermodynamics also means that the system will tend to equilibrium spontaneously. This requires that without perturbation, a system in equilibrium should remain in equilibrium. For heat conduction problems, it means that if the boundary and initial conditions are all in equilibrium, the whole temperature field must maintain temperature equilibrium. For Fourier's law, this requirement by the second law of thermodynamics can be mathematically understood as follows: if the boundary conditions $\left.T\right|_{\Gamma}$ and the initial conditions $\left.T\right|_{t=0}$ satisfy $\left.T\right|_{\Gamma}=\left.T\right|_{t=0}=C_{0}$, where $C_{0}$ is a constant, the whole temperature $T(x, y, z, t)$ must satisfy $T(x, y, z, t)=C_{0}$. For the $C V$ model, the heat conduction equation contains a second-order time derivative term. Therefore, we need two initial conditions $\left.T\right|_{t=0}$ and $\left.\frac{\partial T}{\partial t}\right|_{t=0}$ to determine the solution for the CV model. Then the equilibrious boundary and initial conditions for the CV model are $\left.T\right|_{\Gamma}=\left.T\right|_{t=0}=C_{0}$ and $\left.\frac{\partial T}{\partial t}\right|_{t=0}$. If these conditions are satisfied, the whole temperature field must be $C_{0}$. From the view point of physics, it is not difficult to understand these conclusions. Whether for diffusive transport or for wave transport, if the system has been in equilibrium, equilibrium will not be broken without perturbation. However, for the single-phase-lagging model, the conclusion is different. Even if the boundary and initial conditions are all in equilibrium, equilibrium could be broken in some special circumstances. Next, we will provide a simple example. Consider a one-dimensional problem where the physical properties satisfy $\frac{2 \tau n^{2} \pi}{\rho c_{V} l^{2}}=1$ ( $n$ is an integer). The boundary conditions are taken $\left.T\right|_{x=0, l}=C_{0}$, and the initial condition is taken $\left.T\right|_{t=0}=C_{0}$. Then consider a solution:

$$
T_{\mathrm{I}}(x, t)=C_{1} \sin \frac{n \pi x}{l} \sin \frac{\pi t}{2 \tau}+C_{0}
$$


where $C_{1}$ is an arbitrary constant. This solution satisfies Equation (5), boundary conditions $\left.T\right|_{\Gamma}=\left.T\right|_{t=0, l}=C_{0}$ and initial condition $\left.T\right|_{t=0}=C_{0}$. Obviously, $C_{1} \sin \frac{n \pi x}{l} \sin \frac{\pi t}{2 \tau}\left(C_{1} \neq 0\right)$ breaks equilibrium, and this means that for the SPL model, equilibrium could be broken spontaneously. In this special circumstance, there is no temperature difference at the initial time, but the SPL model could cause a temperature difference. Therefore, for the SPL model, although we can avoid the negative entropy production rate by extending the definition of entropy and proposing appropriate constitutive assumptions, the second law of thermodynamics could also be violated by breaking equilibrium spontaneously. From this view point, for the SPL model, modifying the entropy production rate is not enough to avoid the violation of the second law of thermodynamics. In addition, solution expressed by Equation (22) also shows that the solution for the SPL model may not be unique. For an arbitrary heat conduction problem, where the boundary conditions are taken $\left.T\right|_{x=0, l}=f_{\Gamma}$ and the initial conditions are taken as $\left.T\right|_{t=0}=f_{0}$, if this problem has a solution $T_{\Lambda}(x, t), T^{\prime}{ }_{\Lambda}(x, t)=T_{\Lambda}(x, t)+C_{1} \sin \frac{n \pi x}{l} \sin \frac{\pi t}{2 \tau}$ will also satisfy Equation (5) and all conditions. Therefore, $T^{\prime}{ }_{\Lambda}(x, t)$ is also a solution for this problem, which means that for the SPL model, one determined heat conduction problem could have infinitely many solutions. It is necessary to emphasize that these above non-physical phenomena only occur with special physical properties $\frac{2 \tau n^{2} \pi}{\rho c_{V} l^{2}}=1$.

To avoid the above non-physical problems caused by the SPL model, we provide two assumptions from the view point of engineering and physics. One assumption is that heat conduction process begins at $t=0$, and the system is in thermal equilibrium when $t<0$. Another assumption is that the whole temperature field is finite. From the first assumption, we have $T(x, y, z, t)=C_{2}$ when $t<0$. Let $\varphi=T-C_{2}$ and from Equation (5), $\varphi$ satisfies:

$$
\frac{\partial \varphi}{\partial t}=\frac{\lambda}{\rho c_{V}} \nabla^{2} \varphi(t-\tau)
$$

The boundary and initial conditions of $\varphi$ are $\left.\varphi\right|_{\Gamma}=\left.T\right|_{\Gamma}-C_{2}$ and $\left.\varphi\right|_{t=0}=\left.T\right|_{t=0}-C_{2}$. From the second assumption, we can obtain that $\varphi$ is also finite, and the Laplace integral $F=\int_{0}^{+\infty} \varphi e^{-p t} d t$ is convergent. Therefore, the Laplace transform $F=\int_{0}^{+\infty} \varphi e^{-p t} d t$ exists. Substituting it into Equation (23), and according to the Time-Shift Theorem, we can obtain:

$$
\frac{\lambda}{\rho c_{V}} e^{-p \tau} \nabla^{2} F=p F-\left.\varphi\right|_{t=0}
$$

Equation (24) is an elliptic differential equation whose solution is unique, and therefore, $F=\int_{0}^{+\infty} \varphi e^{-p t} d t$ is unique. What's more, $\varphi$ must be continuous in time because there is a time differential $\frac{\partial \varphi}{\partial t}$ in Equation (23). Based on the continuity of $\varphi$, we can determine that $\varphi$ is unique from Lerch's Theorem. Therefore, based on the two assumptions, the solution for the SPL model will be unique. Then, considering the above problem where $\left.T\right|_{\Gamma}=\left.T\right|_{t=0}=C_{0}$, it is not difficult to find that $T_{\mathrm{II}}(x, t)=C_{0}$ is a solution for Equation (5) with these conditions, and because of the uniqueness, there is no other solution besides $C_{0}$. Then, we conclude that the two assumptions can avoid breaking equilibrium spontaneously.

\section{Mathematical Energy Integral}

Mathematical solution in Section 4 shows that the SPL model could break equilibrium spontaneously. We will prove that Fourier's law and the CV model cannot break equilibrium spontaneously by analyzing mathematical energy integral. Due to Fourier's law and the equilibrious boundary conditions $\left.T\right|_{\Gamma}=C_{0}$, the definition of energy integral is:

$$
E_{F}(t)=\iiint \frac{1}{2}\left(T-C_{0}\right)^{2} d V
$$


which shows the deviation from equilibrium and the vibration amplitude from the equilibrium temperature $C_{0}$. The changing rate of the energy integral can be written as:

$$
\frac{d E_{F}(t)}{d t}=-\iiint \frac{\lambda}{\rho c_{V}}(\nabla T)^{2} d V
$$

which is negative or zero. $\frac{d E_{F}(t)}{d t} \leq 0$ shows why Fourier heat conduction processes tend to equilibrium spontaneously from the view point of mathematics. When $\left.T\right|_{t=0}=C_{0}$, we can obtain $E_{F}(0)=0$, and from $\frac{d E_{F}(t)}{d t} \leq 0, E_{F}(t) \leq E_{F}(0)=0$. However, from Equation (25), obviously $E_{F}(t) \geq 0$, and then we have $0 \leq E_{F}(t) \leq 0$. Therefore, we can obtain $E_{F}(t)=0$ and $T(x, y, z, t)=C_{0}$. This shows that Fourier heat conduction will not break equilibrium when the boundary and initial conditions are all in equilibrium. For the CV model, the definition of energy integral is:

$$
E_{C V}(t)=\iiint\left[\left(\frac{\partial T}{\partial t}\right)^{2}+\frac{\lambda}{\rho c_{V} \tau}(\nabla T)^{2}\right] d V
$$

The rate of change of the energy integral is:

$$
\frac{d E_{C V}(t)}{d t}=-\frac{2}{\tau} \iiint\left(\frac{\partial T}{\partial t}\right)^{2} d V
$$

which is also negative or zero. When $\left.\frac{\partial T}{\partial t}\right|_{t=0}=0$ and $\left.T\right|_{t=0}=C_{0}$, we can obtain $\left.\nabla T\right|_{t=0}=0$ and then we have $E_{C V}(0)=0$. Equation (28) shows that $\frac{d E_{C V}(t)}{d t} \leq 0$, and therefore, $E_{C V}(t) \leq E_{C V}(0)=0$. From Equation (27), obviously $E_{C V}(t) \geq 0$, and then we have $0 \leq E_{C V}(t) \leq 0$. Therefore, we can obtain $E_{C V}(t)=0$, which shows that $\nabla T(x, y, z, t)=0$ and $\frac{\partial T(x, y, z, t)}{\partial t}=0$. Because $\frac{\partial T(x, y, z, t)}{\partial t}=0$ and $\nabla T(x, y, z, t)=0$, we can finally obtain $T(x, y, z, t)=C_{0}$. Thus, it is shown that the CV model will not break equilibrium when the boundary and initial conditions are all in equilibrium. For the CV model, the energy integral Equation (27) doesn't show the vibration amplitude from the equilibrium temperature but the size of the first-order differentials. In Equation (26), we find that the decay rate of $E_{F}(t)$ is determined by $(\nabla T)^{2}$, which means the attenuation will not stop unless $\nabla T=0$. Then, we have $q=0$ and $T(x, y, z, t)=C_{0}$. Therefore, for Fourier's law, the attenuation of the heat conduction process will not stop until the equilibrium is achieved, when $E_{F}(t)=0$. As comparison, in Equation (28), the decay rate of $E_{C V}(t)$ is determined by $\left(\frac{\partial T}{\partial t}\right)^{2}$, which means the attenuation will not stop unless $\frac{\partial T}{\partial t}=0$. Different from Fourier's law, for the CV model, when the attenuation stops, it is possible that $\nabla T \neq 0$, $q \neq 0, T(x, y, z, t) \neq C_{0}$ and $\frac{\partial q}{\partial t} \neq 0$. As an example, for $T(x, y, z, t) \neq C_{0}, \nabla T \neq 0$ and $q \neq 0, T(x, t)=k x+b$ and $q(x, t)=-\lambda k x$ could satisfy the CV model and heat conduction equation Equation (3), where $k$ and $b$ are constants. As an example for $\frac{\partial q}{\partial t} \neq 0, T(x, t)=C_{0}$ and $q(x, t)=q_{0} e^{-\frac{t}{\tau}}$ could satisfy the CV model and heat conduction equation Equation (3), where $q_{0}$ is constant. Therefore, for the CV model, the attenuation of the heat conduction process stops as long as the temperature achieves stability, and it is possible that $\nabla T \neq 0, q \neq 0, T(x, y, z, t) \neq C_{0}$ and $\frac{\partial q}{\partial t} \neq 0$. In summary, from the analysis of mathematical energy integral Fourier's law means that heat conduction must tend to equilibrium, and the $\mathrm{CV}$ model only requires that heat conduction tends to steady temperature.

\section{Conclusions}

In this paper, thermodynamics problems for the SPL model are discussed mainly in terms of the violation of the second law of thermodynamics. The major conclusions about these problems are as follows:

1. In the framework of classical irreversible thermodynamics, the SPL model could cause a negative entropy production rate problem. There are two perspectives for the SPL model to avoid the negative entropy production rate. One is extending the definition of entropy in classical 
irreversible thermodynamics, which is based on extended irreversible thermodynamics. The other is reducing the thermal relaxation time, which is based on the continuity of heat flux in time.

2. It is shown that modifying the entropy production rate to a positive or zero value is not enough to avoid the violation of the second law of thermodynamics for the SPL model, because the SPL model could cause spontaneous equilibrium breaking under some special circumstances. What's more, the SPL model could also lead to infinitely many solutions for determined heat conduction problems, which is also non-physical. To avoid these problems, two assumptions are proposed from the view point of engineering and physics. One assumption is that heat conduction process begins at $t=0$, and the system is in thermal equilibrium when $t<0$. The other assumption is that the whole temperature field is finite.

3. It is proved that Fourier's law and the CV model cannot break equilibrium spontaneously by analyzing the mathematical energy integral. The energy integral of Fourier's law shows the deviation from equilibrium and the vibration amplitude from the equilibrium temperature, and the energy integral of the CV model shows the size of the first-order differentials. For Fourier's law, the attenuation of the heat conduction process will not stop until the equilibrium is achieved, but for the CV model, the attenuation of the heat conduction process stops as long as the temperature achieves stability.

Acknowledgments: This work was supported by the National Natural Science Foundation of China (Grant Nos. 51322603, 51676108, 51136001, 51356001), Science Fund for Creative Research Groups (No. 51321002).

Author Contributions: Shu-Nan Li derived the mathematical models and drafted the paper; Bing-Yang Cao is in charge of the projects and commented the paper.

Conflicts of Interest: The authors declare no conflict of interest.

\section{References}

1. Qiu, T.Q.; Tien, C.L. Short-pulse laser heating on metals. Int. J. Heat Mass Transf. 1992, 35, 719-726. [CrossRef]

2. Joseph, D.D.; Preziosi, L. Heat waves. Rev. Modern Phys. 1989, 61, 41-73. [CrossRef]

3. Joseph, D.D.; Preziosi, L. Addendum to the paper "Heat waves". Rev. Modern Phys. 1990, 62, 375-391. [CrossRef]

4. Chester, M. Second sound in solids. Phys. Rev. 1963, 131, 2013-2015. [CrossRef]

5. Sellitto, A.; Cimmelli, V.A.; Jou, D. Analysis of three nonlinear effects in a continuum approach to heat transport in nanosystems. Physica D 2012, 241, 1344-1350. [CrossRef]

6. Shen, B.; Zhang, P. Thermoacoustic wave propagation and reflection near the liquid-gas critical point. Phys. Rev. E 2009, 79, 060103.

7. Cattaneo, C. Sur une forme de léquation de lachaleur éliminant le paradoxe d'une propagation instantanée. C. R. Acad. Sci. Paris 1958, 247, 431-433.

8. Vernotte, P. Les paradoxes de la théorie continue de l'équation de la chaleur. C. R. Acad. Sci. Paris 1958, 246, 3154-3155.

9. Taitel, Y. On the parabolic, hyperbolic and discrete formulation of the heat conduction equation. Int. J. Heat Mass Transf. 1972, 15, 369-371. [CrossRef]

10. Barletta, A.; Zanchini, E. Unsteady heat conduction by internal-energy waves in solids. Phys. Rev. B 1997, 55, 14208-14213. [CrossRef]

11. Zanchini, E. Hyperbolic heat-conduction theories and nondecreasing entropy. Phys. Rev. B 1999, 60, 991-997. [CrossRef]

12. Sharma, K.R. Damped Wave Transport and Relaxation; Elsevier: Amsterdam, The Netherlands, 2005.

13. Fabrizio, M.; Franchi, F. Delayed thermal models: Stability and thermodynamics. J. Therm. Stress 2014, 37, 160-173. [CrossRef]

14. Fabrizio, M.; Lazzari, B. Stability and second law of thermodynamics in dual-phase-lag heat conduction. Int. J. Heat Mass Transf. 2014, 74, 484-489. [CrossRef]

15. Jou, D.; Casas-Vazquez, J.; Lebon, G. Extended Irreversible Thermodynamics; Springer: Berlin, Germany, 2010.

16. Tzou, D.Y. Thermal shock phenomena under high-rate response in solids. Annu. Rev. Heat Transf. 1992, 4, 111-185. [CrossRef] 
17. Cheng, L.; Xu, M.T.; Wang, L.Q. From Boltzmann transport equation to single-phase-lagging heat conduction. Int. J. Heat Mass Transf. 2008, 51, 6018-6023. [CrossRef]

18. Xu, M.T.; Guo, J.F.; Wang, L.Q.; Cheng, L. Thermal wave interference as the origin of the overshooting phenomenon in dual-phase-lagging heat conduction. Int. J. Therm. Sci. 2011, 50, 825-830. [CrossRef]

19. Xu, M.T.; Wang, L.Q. Dual-phase-lagging heat conduction based on Boltzmann transport equation. Int. J. Heat Mass Transf. 2005, 48, 5616-5624. [CrossRef]

20. Quintanilla, R.; Racke, R. A note on stability in three-phase-lag heat conduction. Int. J. Heat Mass Transf. 2008, 51, 24-29. [CrossRef]

21. Shen, B.; Zhang, P. Notable physical anomalies manifested in non-Fourier heat conduction under the dual-phase-lag model. Int. J. Heat Mass Transf. 2008, 51, 1713-1727. [CrossRef]

22. Li, S.N.; Cao, B.Y. On defects of Taylor series approximation in heat conduction models. Int. J. Heat Mass Transf. 2016, 98, 824-832. [CrossRef]

23. Anisinov, S.I.; Kapeliovich, B.L.; Perelman, T.L. Electron emission from metal surfaces exposed to ultrashort laser pulses. J. Exp. Theor. Phys. 1974, 39, 375-377.

24. Guyer, R.A.; Krumhansl, J.A. Solution of the linearized phonon Boltzmann equation. Phys. Rev. 1966, 148, 766-778. [CrossRef]

25. Tzou, D.Y. A unified field approach for heat conduction from macro- to micro-scales. ASME J. Heat Transf. 1995, 117, 8-16. [CrossRef]

26. Guo, Z.Y.; Hou, Q.W. Thermal wave based on the thermomass model. ASME J. Heat Transf. 2010, 132, 072403. [CrossRef]

27. Sellitto, A.; Cimmelli, V.A. A continuum approach to thermomass theory. ASME J. Heat Transf. 2012, 134, 112402. [CrossRef]

28. Cao, B.Y.; Guo, Z.Y. Equation of motion of a phonon gas and non-Fourier heat conduction. J. Appl. Phys. 2007, 102, 053503. [CrossRef]

29. Dong, Y.; Cao, B.Y.; Guo, Z.Y. Generalized heat conduction laws based on thermomass theory and phonon hydrodynamics. J. Appl. Phys. 2011, 110, 063504. [CrossRef]

(C) 2016 by the authors; licensee MDPI, Basel, Switzerland. This article is an open access article distributed under the terms and conditions of the Creative Commons Attribution (CC-BY) license (http://creativecommons.org/licenses/by/4.0/). 